\title{
A dietary cholesterol challenge study to assess Chlorella supplementation in maintaining healthy lipid levels in adults: a double-blinded, randomized, placebo- controlled study
}

\author{
Sangmi Kim ${ }^{1+}$, Joohee Kim ${ }^{1 \dagger}$, Yeni $\mathrm{Lim}^{1}$, You Jin Kim ${ }^{1}$, Ji Yeon $\mathrm{Kim}^{2}$ and Oran Kwon ${ }^{1 *}$
}

\begin{abstract}
Background: Previous animal studies suggested that Chlorella, a unicellular green alga, has a preventive role in maintaining serum cholesterol levels against excess dietary cholesterol intake. This study aimed to conduct a pioneering investigation to clarify this issue in healthy subjects by adopting a dietary cholesterol challenge, which has not been used previously in similar studies of Chlorella in hypercholesterolemia.

Methods: In this double blind, randomized, placebo-controlled study, 34 participants ingested $510 \mathrm{mg}$ of dietary cholesterol from three eggs concomitantly with a usual dose of Chlorella $(5 \mathrm{~g} / \mathrm{d})$ or a matched placebo for 4 weeks.

Results: The dietary cholesterol challenge induced consistently higher concentrations of serum total cholesterol (TC, $P<0.001)$, LDL-C $(P=0.004)$, and HDL-C $(P=0.010)$ compared with baseline values, suggesting that the challenge was reliable. Thus, we observed a preventive action of Chlorella in maintaining serum TC versus placebo levels ( $3.5 \%$ versus $9.8 \%$, respectively; $P=0.037)$ and LDL-C versus placebo levels $(1.7 \%$ versus $14.3 \%$, respectively; $P=0.012$ ) against excessive dietary cholesterol intake and in augmenting HDL-C versus placebo levels ( $8.3 \%$ versus $3.8 \%$, respectively). Furthermore, serum a-carotene showed the best separation between the placebo and Chlorella groups $\left(R^{2} X\right.$ and $\left.R^{2} Y>0.5 ; Q^{2}>0.4\right)$.

Conclusion: The results suggest that a fully replicated dietary cholesterol challenge may be useful in assessing the effectiveness of dietary supplements in maintaining the serum lipid profiles of adults whose habitual diets are high in cholesterol.
\end{abstract}

Trial registration: WHO International Clinical Trials Registry Platform (KCT0000258)

Keywords: Carotenoids, Chlorella, Dietary cholesterol challenge, Healthy adult

\section{Background}

Cardiovascular disease (CVD) is the leading cause of mortality worldwide as a result of the aging population and increased urbanization. A high serum cholesterol level is thought to be a major risk factor for CVD [1]. Each $1 \%$ increase in the serum cholesterol is predicted

* Correspondence: orank@ewha.ac.kr

${ }^{\dagger}$ Equal contributors

${ }^{1}$ Department of Nutritional Science and Food Management, Ewha Womans University, Seoul 03760, Republic of Korea

Full list of author information is available at the end of the article to increase the risk of coronary disease by approximately $2 \%$ [2]. An impressive body of evidence has accumulated regarding the hypocholesterolemic effects associated with several plant-based foods compared with the placebo group in at risk subjects such as those with mild to severe hypercholesterolemia [3] or compared before and after administration in healthy subjects [4]. In animal models, a preventive effect of a food or food ingredient against hypercholesterolemia could be shown by the co-administration of excess dietary cholesterol in normal animals [5]. However, no successful 
placebo-controlled clinical trial has yet been explored to validate this concept in human subjects.

Chlorella is a unicellular green alga that contains a wide array of nutrients, including carotenoids, chlorophyll, minerals, vitamins and long-chain polyunsaturated fatty acids [5]. Animal experiments have shown that Chlorella inhibited the intestinal absorption of excess cholesterol from the diet and enhanced fecal steroid excretion, thereby preventing hypercholesterolemia [6]. However, several previous human clinical trials, including our own, have attempted only to determine only whether Chlorella supplementation is useful to reduce the high cholesterol levels of hypercholesterolemic subjects [7].

Here, we performed a double-blinded, randomized, placebo-controlled trial to investigate the hypothesis that a dietary cholesterol challenge might be useful to evaluate the effectiveness of Chlorella supplementation in maintaining healthy serum cholesterol concentration in adults consuming a high cholesterol meal. This approach is based on the previous findings of other investigations, which indicated that consuming three eggs daily could increase serum cholesterol levels in healthy subjects $[8,9]$. Furthermore, we examined whether carotenoids derived from the eggs may have hampered the prediction of the responses of serum lipids to Chlorella carotenoids by using partial least squares (PLS) regression.

\section{Methods}

\section{Test samples}

Tablets containing $416 \mathrm{mg}$ of dried Chlorella (Chlorella vulgaris) or a color-matched placebo (lactose) were kindly provided by Daesang Corp. (Seoul, Korea). Chlorella is a unicellular green alga and listed as a functional ingredient in the Korean Health/Functional Food Code with the following specifications: total chlorophyll content $>10 \mathrm{mg} / \mathrm{g}$ for identification; and lead < $3.0 \mathrm{mg} / \mathrm{kg}$, cadmium $<1.0 \mathrm{mg} / \mathrm{kg}$, total mercury $<0.5 \mathrm{mg} /$ $\mathrm{kg}$, total pheophorbide $<1,000 \mathrm{mg} / \mathrm{kg}$, and coliform bacteria negative for purity. Daily Chlorella supplementation (12 tablets/d) provides $5 \mathrm{~g}$ of Chlorella containing the following nutrients: $3 \mathrm{~g}$ of protein, $180 \mathrm{mg}$ of total carbohydrates, $640 \mathrm{mg}$ of total lipids, $650 \mathrm{mg}$ of dietary fiber, $225 \mathrm{mg}$ of ash, 2,945 IU of vitamin A, $3.7 \mathrm{mg}$ of vitamin $C$, and $1.1 \mathrm{mg}$ of vitamin E [6]. The dietary source of the cholesterol challenge were pasteurized whole eggs were purchased from a retail market in Seoul, Korea. Daily egg consumption (3 eggs/d) provides $540 \mu \mathrm{g}$ of lutein, $330 \mu \mathrm{g}$ of zeaxanthin, $10 \mu \mathrm{g}$ of $\beta$ carotene and trace amount of $\alpha$-carotene, and $510 \mathrm{mg}$ cholesterol.

\section{Study design}

The double blind, randomized, and placebo-controlled study was performed in two phases, a 4-week lead-in phase and a 4-week intervention phase. The length of the intervention phase was determined based on a metaanalysis of plant sterols as cholesterol lowering agents [10]. To detect the postulated difference with $80 \%$ power using a two-sided $5 \%$, and a $20 \%$ dropout rate, the sample size was estimated to be 34 subjects. The study protocol was approved by the Institutional Review Boards of Ewha Womans University (Seoul, Korea) and registered at the WHO International Clinical Trials Registry Platform as KCT0000258. The study was conducted in accordance with the Declaration of Helsinki, and the results are reported according to the Consolidated Standards of Reporting Trials guidelines [11].

\section{Subjects}

After the initial screening, 34 eligible subjects were entered at baseline. For study inclusion, a serum total cholesterol (TC) levels of $<5.18 \mathrm{mmol} / \mathrm{L}$ (desirable level) [12] was required of the subjects at the screening visit. The following exclusion criteria were applied: the regular use of medications or dietary supplements; presence of CVD, hypertension, type 2 diabetes, liver disease, renal failure, thyroid disease, or malignant tumors; a family history of hypercholesterolemia; known hypersensitivity to the study products; and pregnancy or lactation. All of the participants provided written informed consent before participation.

\section{Study procedures}

Following the 4-week lead-in period, the participants were randomly assigned to either the placebo or Chlorella group. At the beginning of the study, the participants received one bottle of the test sample and 21 eggs (packaged in threes for a daily supply), enough for seven days, thus allowing for weekly visits. They were asked to take four tablets of the test sample and one egg with each meal, for a total of $5 \mathrm{~g}$ of Chlorella powder and $510 \mathrm{mg}$ of cholesterol daily. Cooking methods for the eggs were provided to thereby minimize influences on carotenoid absorption. To determine a compliance status, the subjects were asked to answer whether they had followed the dietary instruction and the remaining pills were counted at each visit. The subjects were also required to report any possible adverse events. To assess nutrient intake and monitor dietary compliance, three-day dietary records were analyzed using a computer aided nutritional analysis program (Can-Pro 3.0, the Korean Nutrition Society, Seoul, Korea).

\section{Outcome measurements}

At the beginning of the study and 4 weeks after the intervention, overnight fasting blood was collected in serum separator tubes (Becton Dickinson, Franklin Lakes, NJ, USA). The samples were then centrifuged, 
and the supernatants were stored at $-80{ }^{\circ} \mathrm{C}$ before analysis. The serum TC, triglycerides (TG), and highdensity lipoprotein cholesterol (HDL-C) concentrations were assessed using commercially available kits (Daiichi Pure Chemicals, Tokyo, Japan). The low-density lipoprotein cholesterol (LDL-C) level was calculated using the Friedewald equation (LDL-C $=\mathrm{TC}-\mathrm{HDL}-\mathrm{C}-\mathrm{TG}$ / 2.2). Serum carotenoid concentrations were measured by HPLC (Shiseido SP 3023, Tokyo, Japan) with a reverse-phase C18 column (Shiseido Capcell Pak, MG type, $5 \mu \mathrm{m}, 4.6 \times 250 \mathrm{~mm}$ ) and UV detector.

\section{Statistical analysis}

Data analyses were performed on the intention-to-treat (ITT) population, which included all randomized subjects. Additionally, per protocol (PP) analysis was performed on participants who completed the study. The results of the ITT and PP analyses were comparable, thus only those of the ITT analysis are shown. Variables were tested for normal distribution using the ShapiroWilk $W$-test and $\log$ transformation was performed on skewed variables. A Student's $t$-test or Fisher's exact test was used to compare the baseline characteristics between groups. An analysis of variance (ANOVA) mixed-effects model was applied to compare changes in nutrient intake, serum lipids, and serum carotenoids with group and time as fixed effects factors and subject as random effect factor. A two-tailed value of $P<0.05$ was considered to be significantly different. PLS regression was used to correlate measured values of serum carotenoids for serum lipids and identify predictors for responses to Chlorella during the dietary cholesterol challenge. PLS regression with 5-fold cross-validation leads to the calculation of the $R^{2} X, R^{2} Y$, and $Q^{2}$ factors, representing the explanation, fitness, and prediction power, respectively. $R^{2}>0.5$ and $\mathrm{Q}^{2}>0.4$ [13] were considered acceptable for PLS regression. The data were analyzed using the SAS 9.3 statistical software (SAS Institute, Cary, NC, USA) or TANAGRA (version1.4.50, University of Lyon, France).

\section{Results}

\section{Subject characteristics and dietary monitoring}

The CONSORT flow chart of the study is shown in Fig. 1. Following the 4-week lead-in period, 34 eligible subjects were randomized into two groups. Five subjects were withdrawn from the study due to lack of interest $(n=1)$ or loss of contact $(n=4)$. The compliance was excellent (mean compliance ratio for all subjects $=97.5 \%$ ) with no subjects were excluded due to insufficient compliance. No participant reported any significant subjective symptoms or serious events.

The participant characteristics at baseline are shown in Table 1. All subjects were in good health, as determined by physical examination and routine blood and urine biochemical screening. Randomization was successful, and no significant differences were observed in the baseline findings between the groups. The daily intakes of calories and some selected nutrients at baseline and week 4 are listed in Table 2. Within each group, cholesterol intake increased by approximately 3 -fold by adding three eggs to the usual diet $(P<0.001$ for time effect). No significant between-group differences were observed for any nutrient, however, $\beta$-carotene intake was significantly increased in the Chlorella group compared to the placebo group ( $P=0.010$ for interaction). Unexpectedly, carbohydrate intake was decreased in both groups ( $P=0.033$ for time effect).

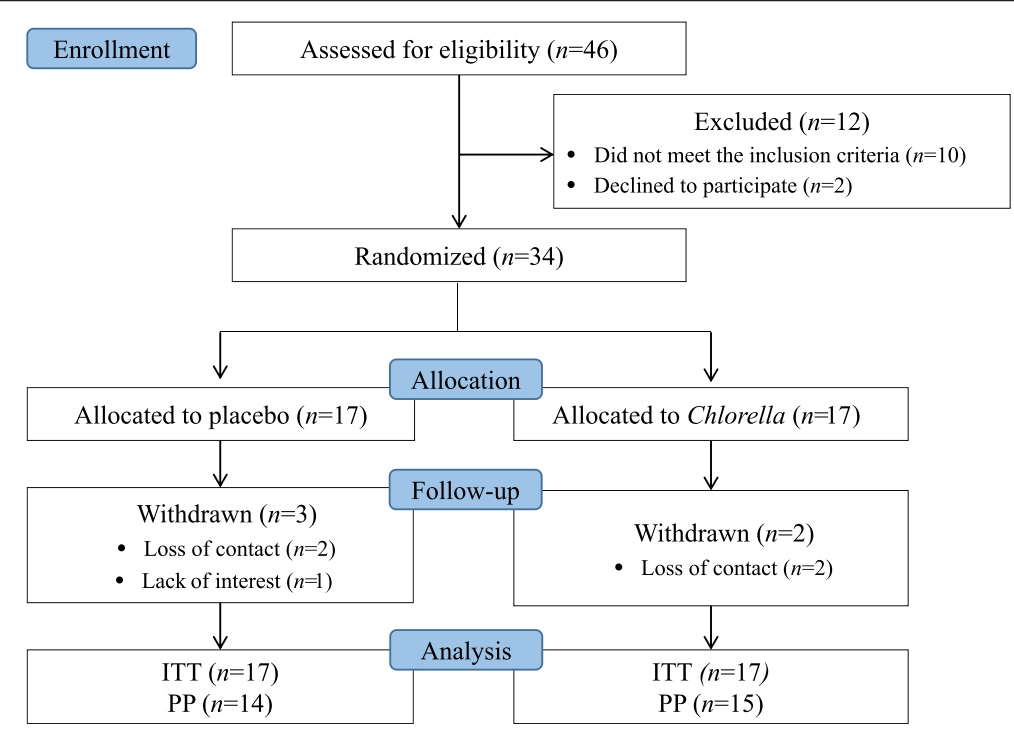

Fig. 1 CONSORT diagram for the flow of subjects through the study. ITT, intention-to-treat; PP, per protocol 
Table 1 Characteristics of the participants at baseline

\begin{tabular}{llll}
\hline & $\begin{array}{l}\text { Placebo group } \\
(n=17)\end{array}$ & $\begin{array}{l}\text { Chlorella group } \\
(n=17)\end{array}$ & $P$-value \\
\hline Age, y & $23.7 \pm 0.8$ & $23.2 \pm 0.6$ & 0.639 \\
Female/Male, $n$ & $15 / 2$ & $15 / 2$ & 1.000 \\
BMl, $\mathrm{kg} / \mathrm{m}^{2}$ & $19.7 \pm 0.5$ & $20.7 \pm 0.4$ & 0.130 \\
$\mathrm{SBP}, \mathrm{mmHg}$ & $106.4 \pm 2.4$ & $104.4 \pm 2.3$ & 0.557 \\
DBP, $\mathrm{mmHg}$ & $63.8 \pm 1.9$ & $64.6 \pm 1.7$ & 0.737 \\
Fasting serum levels, $\mathrm{mmol} / \mathrm{L}$ & & \\
$\mathrm{TC}$ & $4.24 \pm 0.16$ & $4.38 \pm 0.12$ & 0.495 \\
$\mathrm{TG}$ & $0.76 \pm 0.07$ & $0.83 \pm 0.08$ & 0.678 \\
LDL-C & $2.28 \pm 0.11$ & $2.49 \pm 0.11$ & 0.186 \\
HDL-C & $1.58 \pm 0.08$ & $1.55 \pm 0.06$ & 0.737 \\
\hline
\end{tabular}

Values are the means \pm SEMs (all such values). $P$-values are for Student's $t$-test or Fisher's exact test used to compare the between-group differences. $B M I$ body mass index, SBP systolic blood pressure, DBP diastolic blood pressure, $T C$ total cholesterol, TG triglycerides, LDL-C low-density lipoprotein cholesterol, HDL-C high-density lipoprotein cholesterol

The role of Chlorella in maintaining health serum lipid profiles following a dietary cholesterol challenge

No difference in baseline concentrations of serum lipids was observed between the two groups. After 4 weeks, the 3-egg dietary cholesterol challenge induced significant increases in serum TC $(P<0.001$ for time effect), LDL-C $(P=0.004$ for time effect), and HDL-C ( $P=0.010$ for time effect) in the placebo group. In contrast, concomitant ingestion of $5 \mathrm{~g}$ Chlorella significantly suppressed the increases of TC $(9.8 \%$ versus $3.5 \% ; P=0.037$ for interaction) and LDL-C (14.3\% versus $1.7 \% ; P=0.012$ for interaction), but accelerated the increase of HDL-C (3.8\% versus $8.3 \%$ ), a finding that lacked statistical significance (Table 3, upper part).

\section{Serum carotenoids as a predictor of serum lipid responses to Chlorella supplementation}

The daily ingestion of three eggs resulted in significant increases in the serum lutein, $\alpha$-carotene and $\beta$-carotene levels $(P<0.001$ for time effect for all) in the placebo group. Concomitant intake of Chlorella supplementation led to a significant enhancement of lutein (76\% versus $123 \% ; P=0.006$ for interaction) and $\alpha$-carotene $(31.2 \%$ versus $228 \%, P<0.001$ for interaction) levels. However, serum $\beta$-carotene concentration remained unchanged between the two groups (Table 3, lower part).

PLS regressions for each combination of serum carotenoids and lipids showed that data points from $\beta$ carotene (Fig. 2a) and lutein (Fig. 2b) were randomly dispersed in the plane and did not show clear discrimination between groups. In contrast, $\alpha$-carotene showed better separations between the placebo and Chlorella groups $\left(R^{2} X\right.$ and $R^{2} Y>0.5 ; Q^{2}>0.4$ ) (Fig. 2c). The finding suggests that $\alpha$-carotene may serve an important parameter for predicting the maintenance effect of Chlorella on healthy cholesterol concentrations.

\section{Discussion}

The data obtained in this study validated the hypothesis that a dietary cholesterol challenge permitted the determination of a beneficial impact of Chlorella on modifying serum cholesterol concentration in subjects whose habitual diets are high in cholesterol. Early changes in metabolic markers may not be easily identified due to the robustness of homeostatic vigor of healthy subjects [14]. The administration of additional dietary cholesterol from three eggs daily created a temporary perturbation in serum cholesterol levels, thus providing a unique opportunity to evaluate the intervention effect of Chlorella on the earliest changes in

Table 2 Mean daily intakes of selected nutrients at baseline and during the 4-week intervention

\begin{tabular}{|c|c|c|c|c|c|c|c|}
\hline \multirow[t]{2}{*}{ Nutrients } & \multicolumn{2}{|c|}{ Placebo group $(n=17)$} & \multicolumn{2}{|c|}{ Chlorella group $(n=17)$} & \multicolumn{3}{|c|}{$P$-value ${ }^{a}$} \\
\hline & Week 0 & Week 4 & Week 0 & Week 4 & Group & Time & Group $x$ time \\
\hline Energy, kcal/d & $1558 \pm 124$ & $1505 \pm 92$ & $1511 \pm 111$ & $1485 \pm 81$ & 0.800 & 0.642 & 0.860 \\
\hline Carbohydrate, g/d & $204.0 \pm 16.6$ & $185.6 \pm 13.7$ & $214.7 \pm 15.3$ & $186.0 \pm 11.7$ & 0.734 & 0.033 & 0.680 \\
\hline Protein, g/d & $63.0 \pm 5.5$ & $65.3 \pm 3.2$ & $56.2 \pm 7.3$ & $64.9 \pm 3.8$ & 0.400 & 0.065 & 0.394 \\
\hline Fat, g/d & $52.7 \pm 5.1$ & $55.3 \pm 3.3$ & $46.2 \pm 3.5$ & $50.5 \pm 2.5$ & 0.189 & 0.338 & 0.815 \\
\hline SFA, $g / d$ & $8.2 \pm 1.1$ & $9.5 \pm 1.1$ & $7.4 \pm 1.2$ & $9.6 \pm 0.8$ & 0.723 & 0.071 & 0.643 \\
\hline PUFA, $g / d$ & $5.8 \pm 0.7$ & $6.5 \pm 0.8$ & $6.4 \pm 0.9$ & $6.4 \pm 0.5$ & 0.664 & 0.194 & 0.810 \\
\hline Cholesterol, mg/d & $323.5 \pm 54.5$ & $725.9 \pm 17.5$ & $219.1 \pm 32.2$ & $754.1 \pm 25.7$ & 0.312 & $<0.001$ & 0.220 \\
\hline Dietary fibers, g/d & $12.7 \pm 1.3$ & $12.6 \pm 1.1$ & $11.3 \pm 1.1$ & $11.9 \pm 0.6$ & 0.665 & 0.316 & 0.568 \\
\hline$\beta$-carotene, mg/d & $2.2 \pm 0.4$ & $1.7 \pm 0.3$ & $1.9 \pm 0.4$ & $2.6 \pm 0.2$ & 0.266 & 0.302 & 0.010 \\
\hline
\end{tabular}

Values are the means \pm SEMs. Intakes were estimated from 3-day dietary records using CAN-pro (Korean Nutrition Society, Seoul, Korea). Egg, Chlorella and placebo intakes were included in the analysis. SFA saturated fatty acids, PUFA polyunsaturated fatty acids

${ }^{a}$ Mixed-effects ANOVA model, with group and time as fixed effects factors and subject as random effect factor 
Table 3 Serum lipid and carotenoid responses to the 4-week intervention with Chlorella supplementation with three eggs daily

\begin{tabular}{|c|c|c|c|c|c|c|c|}
\hline \multirow{2}{*}{$\begin{array}{l}\text { Fasting serum } \\
\text { levels (mmol/L) }\end{array}$} & \multicolumn{2}{|c|}{ Placebo group $(n=17)$} & \multicolumn{2}{|c|}{ Chlorella group $(n=17)$} & \multicolumn{3}{|c|}{$P$-value ${ }^{a}$} \\
\hline & Week 0 & Week 4 & Week 0 & Week 4 & Group & Time & Group x Time \\
\hline \multicolumn{8}{|l|}{ Serum lipids } \\
\hline $\mathrm{TC}$ & $4.24 \pm 0.16$ & $4.71 \pm 0.15$ & $4.38 \pm 0.12$ & $4.47 \pm 0.12$ & 0.962 & $<0.001$ & 0.037 \\
\hline LDL-C & $2.28 \pm 0.11$ & $2.59 \pm 0.11$ & $2.49 \pm 0.11$ & $2.51 \pm 0.12$ & 0.619 & 0.004 & 0.012 \\
\hline $\mathrm{HDL}-\mathrm{C}$ & $1.58 \pm 0.08$ & $1.67 \pm 0.10$ & $1.55 \pm 0.06$ & $1.62 \pm 0.05$ & 0.917 & 0.010 & 0.404 \\
\hline \multicolumn{8}{|c|}{ Serum carotenoids } \\
\hline Lutein & $0.54 \pm 0.05$ & $0.89 \pm 0.08$ & $0.65 \pm 0.03$ & $1.39 \pm 0.12$ & 0.001 & $<0.001$ & 0.006 \\
\hline a-carotene & $0.06 \pm 0.01$ & $0.07 \pm 0.01$ & $0.08 \pm 0.01$ & $0.24 \pm 0.02$ & $<0.001$ & $<0.001$ & $<0.001$ \\
\hline$\beta$-carotene & $0.19 \pm 0.02$ & $0.27 \pm 0.05$ & $0.22 \pm 0.02$ & $0.29 \pm 0.03$ & 0.292 & $<0.001$ & 0.753 \\
\hline
\end{tabular}

Values are the means \pm SEMs. TC total cholesterol, $L D L$-C low-density lipoprotein cholesterol, HDL-C high-density lipoprotein cholesterol

${ }^{a}$ Mixed-effects ANOVA model with group and time as fixed effects factors and subject as random effect factor

\section{(a) B-carotene}
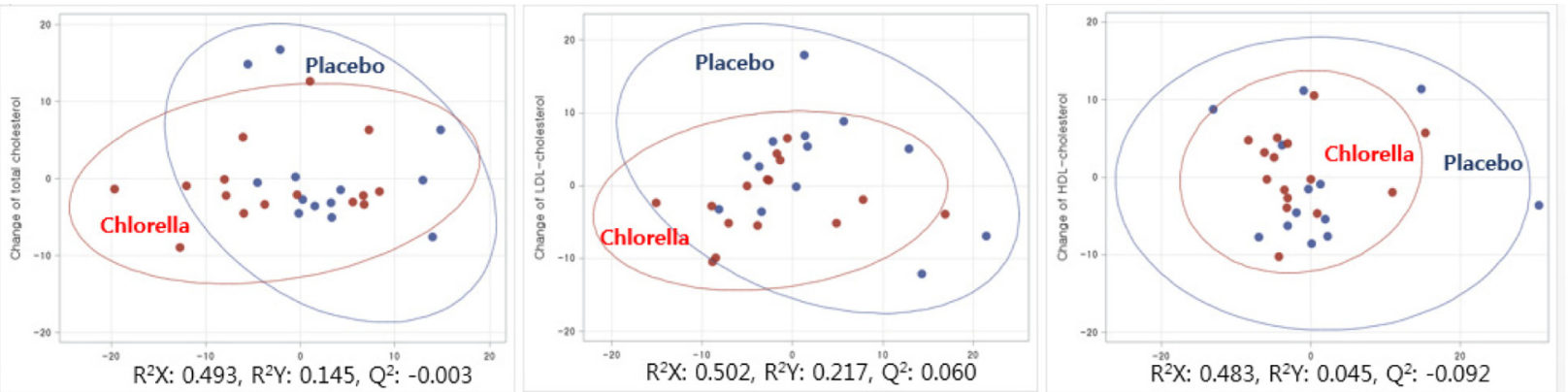

(b) Lutein
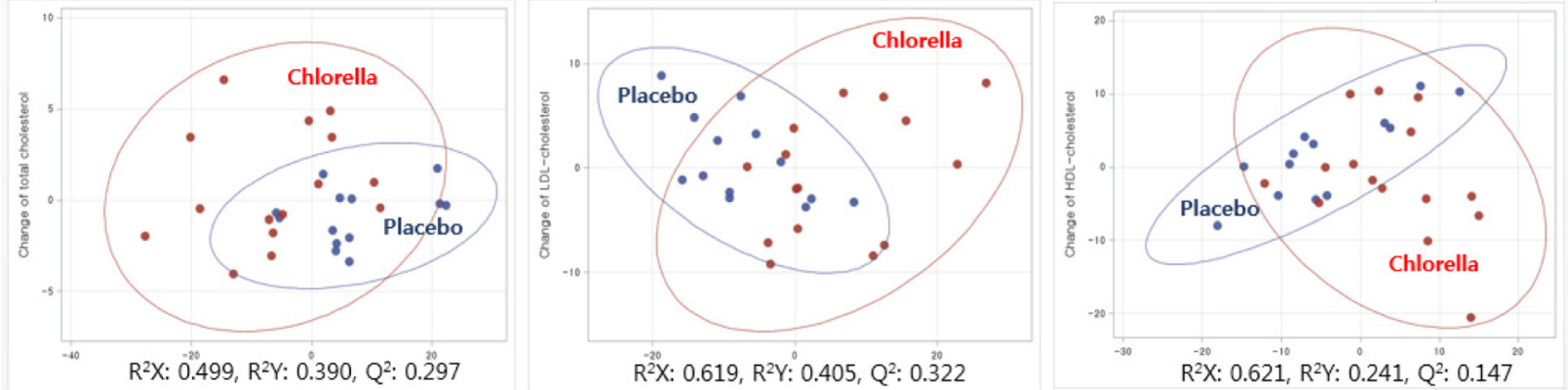

(c) $\alpha$-carotene
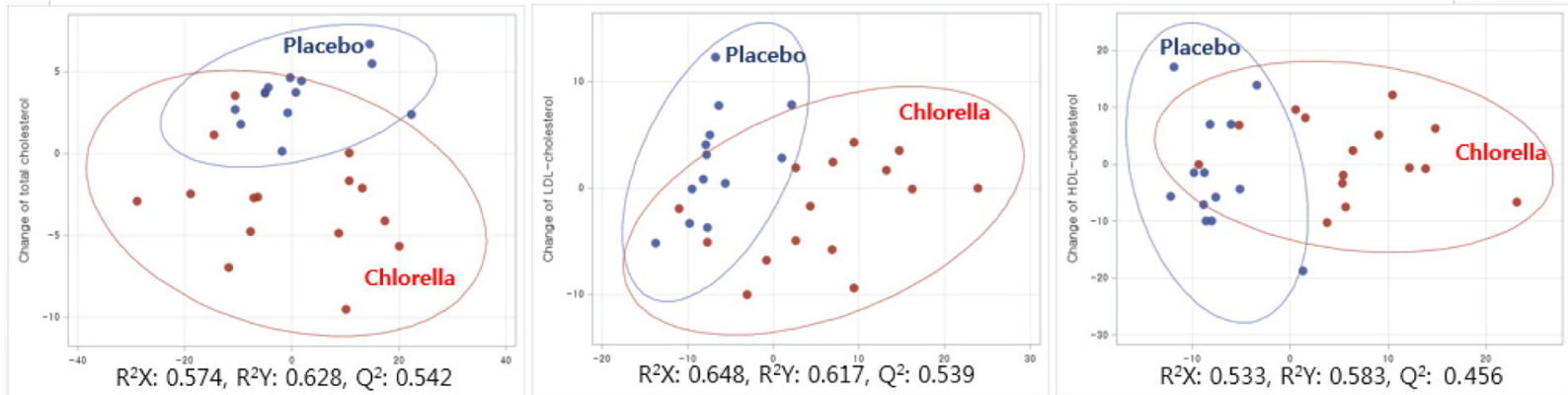

Fig. 2 PLS-regression score plots of each serum lipid and carotenoid obtained from the placebo and Chlorella group. $R^{2} X$, modeled variation of $X$; $R^{2} Y$, predicted variation of $Y$; $Q^{2}$, cross-validated prediction of $Y$. (a) $\beta$-carotene, (b) Lutein, (c) a-carotene 
serum lipid profiles. To the best of our knowledge, this is the first attempt to use a dietary cholesterol challenge to investigate the modifying effect of a food or food component on serum lipid profile in healthy subjects.

Notably, the serum lipid profile changes that ensued from the three egg consumption were modest; (1) the alterations were quantitatively within the normal ranges and the LDL particle subclass was identified as the larger LDL particle [15], which is believed to have a reduced susceptibility to oxidation and endothelial penetration [16]. Furthermore, the $0.40 \mathrm{mmol} / \mathrm{L}$ increase in the serum cholesterol we observed in this study in response to the ingestion of $510 \mathrm{mg}$ of additional cholesterol from three eggs was in agreement with previous studies that estimated a $0.05 \sim 0.12 \mathrm{mmol} / \mathrm{L}$ increase in the serum TC for each additional $100 \mathrm{mg}$ of dietary cholesterol [17, 18]. Together, these results suggest that the 4-week dietary cholesterol challenge from three eggs each day was fully replicated in magnitude the expected changes in serum cholesterol.

Chlorella contains a wide range of bioactive substances that may act to optimize lipid metabolism. In our previous study, we observed that Chlorella carotenoids are highly bioavailable [7, 19]. In this study, we examined whether serum carotenoids derived from Chlorella may be used to predict serum lipid responses to Chlorella consumption during a dietary cholesterol challenge. Because the eggs also contain high level of carotenoids, we first compared the serum carotenoid levels for each group. In the case of lutein, the daily lutein consumption from three eggs $(540 \mu \mathrm{g} / \mathrm{d})$ increased mean serum lutein levels by $58 \%$ at 4 weeks compared to the baseline value. When Chlorella lutein $(7,450 \mu \mathrm{g} /$ d) was added, the mean serum lutein levels increased 2fold compared to egg consumption only. In regard to $\alpha$-carotene, Chlorella provided high levels $(500 \mu \mathrm{g} / \mathrm{d})$, whereas eggs provided trace amounts. The mean serum $\alpha$-carotene level was increased 8-fold following Chlorella supplementation compared to egg consumption only. However, the additional $\beta$-carotene consumption from Chlorella $(900 \mu \mathrm{g} / \mathrm{d})$ was not associated with a significant increase of serum $\beta$-carotene level compared to egg consumption only, likely because of the rapid conversion of $\beta$-carotene to vitamin A [20].

Then, PLS regression analysis was performed to identify the carotenoids that contributed to predicting the effectiveness of Chlorella on serum lipid profile during the dietary cholesterol challenge. Consistent with our previous study in subjects with hypercholesterolemia, the data points from $\alpha$-carotene showed a better separation by PLS regression analysis. Taken together, the cumulative data suggested that serum $\alpha$-carotene concentration might be useful, at least in part, to predict Chlorella effects on serum lipid concentrations during the dietary cholesterol challenge. However, it is a limitation of this study that we did not determine the underlying mechanism that could explain how the results obtained may have been due to the high concentration of serum $\alpha$ carotene. Future studies will be needed to confirm the concomitant effects of carotenoids on cholesterol absorption and to understand the pathways and responses initiated by Chlorella supplementation following a high dietary cholesterol challenge in laboratory animals and human subjects.

\section{Conclusions}

Given the modest nature of the serum lipid response to dietary cholesterol challenge from three eggs daily for 4 weeks, our findings provide an essential foundation for applying a dietary cholesterol challenge can be applied to evaluate candidate foods or food components with hypocholesterolemic properties in healthy subjects. Thereby it is plausible to implicate that Chlorella as playing a useful role in maintaining healthy serum cholesterol levels in the environment of free access to high-lipid foods, thus preventing or delaying the risk for CVD.

\section{Abbreviations}

CVD: cardiovascular disease; HDL-C: high-density lipoprotein cholesterol; HPLC: high performance liquid chromatography; ITT: intention-to-treat; LDL-C: low-density lipoprotein cholesterol; PP: per protocol; TC: total cholesterol; TG: triglycerides.

\section{Competing interests}

The authors declare that they have no competing interests.

\section{Authors' contribution}

OK, JK, and JYK were responsible for the study conception and study design; OK and JK were responsible for the supervision of the human study, sample handling, conduction of the analyses, and statistical analysis; SMK and YL conducted the study and performed analyses of the data; JK and YK performed the statistical analysis; OK and JK prepared the manuscript. All authors read and approved the final manuscript.

\section{Acknowledgments}

We wish to thank the study participants. This study was supported by an unrestricted grant from Daesang Corp. (Seoul, Korea) and the Ministry of Science, ICT \& Future Planning (NRF 2012M3A9C4048761). The funding sources had no involvement in the collection, analysis, and interpretation of the data, the writing of this report, or the decision to submit this manuscript for publication.

\section{Author details}

${ }^{1}$ Department of Nutritional Science and Food Management, Ewha Womans University, Seoul 03760, Republic of Korea. ${ }^{2}$ Department of Food Science and Technology, Seoul National University of Science and Technology, Seoul 01811, Republic of Korea.

Received: 30 January 2016 Accepted: 4 May 2016

Published online: 13 May 2016

\section{References}

1. Wu T, Trevisan M, Genco RJ, Falkner KL, Dorn JP, Sempos CT. Examination of the relation between periodontal health status and cardiovascular risk factors: serum total and high density lipoprotein cholesterol, C-reactive protein, and plasma fibrinogen. Am J Epidemiol. 2000;151:273-82

2. The Lipid Research Clinics Coronary Primary Prevention Trial results. II. The relationship of reduction in incidence of coronary heart disease to cholesterol lowering. JAMA 1984; 251:365-374. 
3. Lu TM, Chiu HF, Shen YC, Chung CC, Venkatakrishnan K, Wang CK. Hypocholesterolemic efficacy of quercetin rich onion juice in healthy mild hypercholesterolemic adults: a pilot study. Plant Foods Hum Nutr. 2015;70: 395-400.

4. Alvarez-Suarez JM, Giampieri F, Tulipani S, Casoli T, Di Stefano G, GonzalezParamas AM, Santos-Buelga C, Busco F, Quiles JL, Cordero MD, et al. One-month strawberry-rich anthocyanin supplementation ameliorates cardiovascular risk, oxidative stress markers and platelet activation in humans. J Nutr Biochem. 2014;25:289-94.

5. Bocanegra A, Bastida S, Benedí J, Ródenas S, Sánchez-Muniz FJ. Characteristics and nutritional and cardiovascular-health properties of seaweeds. J Med Food. 2009;12:236-58.

6. Lee HS, Park HJ, Kim MK. Effect of Chlorella vulgaris on lipid metabolism in Wistar rats fed high fat diet. Nutr Res Pract. 2008;2:204-10.

7. Ryu NH, Lim Y, Park JE, Kim J, Kim JY, Kwon SW, Kwon O. Impact of daily Chlorella consumption on serum lipid and carotenoid profiles in mildly hypercholesterolemic adults: a double-blinded, randomized, placebocontrolled study. Nutr J. 2014;13:57-64.

8. Weggemans RM, Zock PL, Katan MB. Dietary cholesterol from eggs increases the ratio of total cholesterol to high-density lipoprotein cholesterol in humans: a meta-analysis. Am J Clin Nutr. 2001;73:885-91.

9. Fernandez ML. Dietary cholesterol provided by eggs and plasma lipoproteins in healthy populations. Curr Opin Clin Nutr Metab Care. 2006;9:8-12.

10. Abumweis SS, Barake R, Jones PJ: Plant sterols/stanols as cholesterol lowering agents: A meta-analysis of randomized controlled trials. Food Nutr Res 2008, 52 doi: 10.3402/fnr.v52i0.1811

11. Moher D, Hopewell S, Schulz KF, Montori V, Gøtzsche PC, Devereaux PJ, Elbourne D, Egger M, Altman DG, CONSORT. CONSORT 2010 explanation and elaboration: updated guidelines for reporting parallel group randomised trials. Int J Surg. 2012;10:28-55.

12. National Cholesterol Education Program (NCEP) Expert Panel on Detection, Evaluation, and Treatment of High Blood Cholesterol in Adults (Adult Treatment Panel III). Third report of the National Cholesterol Education Program (NCEP) expert panel on detection, evaluation, and treatment of high blood cholesterol in adults (adult treatment panel III) final report. Circulation. 2002;106:3143-421

13. Paban V, Fauvelle F, Alescio-Lautier B. Age-related changes in metabolic profiles of rat hippocampus and cortices. Eur J Neurosci. 2010;31:1063-73.

14. Wopereis S, Rubingh CM, van Erk MJ, Verheij ER, van Vliet T, Cnubben NH, Smilde AK, van der Greef J, van Ommen B, Hendriks HF. Metabolic profiling of the response to an oral glucose tolerance test detects subtle metabolic changes. PLoS One. 2009;4:e4525.

15. Greene CM, Zern TL, Wood RJ, Shrestha S, Aggarwal D, Sharman MJ, Volek JS, Fernandez ML. Maintenance of the LDL cholesterol: HDL cholesterol ratio in an elderly population given a dietary cholesterol challenge. J Nutr. 2005;135:2793-8.

16. Aiver AN, Kip KE, Marroquin OC, Mulukutla SR, Edmundowicz D, Reis SE. Racial differences in coronary artery calcification are not attributed to differences in lipoprotein particle sizes: the Heart Strategies Concentrating on Risk Evaluation (Heart SCORE) study. Am Heart J. 2007;153:328-34.

17. Hegsted DM. Serum-cholesterol response to dietary cholesterol: a re-evaluation. Am J Clin Nutr. 1986;44:299-305.

18. Hopkins PN. Effects of dietary cholesterol on serum cholesterol: a metaanalysis and review. Am J Clin Nutr. 1992;55:1060-70.

19. Jung HY, OK HM, Park MY, Kim JY, Kwon O. Bioavailability of carotenoids from chlorella powder in healthy subjects: A comparison with marigold petal extract. J Funct Foods. 2016;21:27-35.

20. Yeum KJ, Russell RM. Carotenoid bioavailability and bioconversion. Annu Rev Nutr. 2002;22:483-504.

\section{Submit your next manuscript to BioMed Central and we will help you at every step:}

- We accept pre-submission inquiries

- Our selector tool helps you to find the most relevant journal

- We provide round the clock customer support

- Convenient online submission

- Thorough peer review

- Inclusion in PubMed and all major indexing services

- Maximum visibility for your research

Submit your manuscript at www.biomedcentral.com/submit

) Biomed Central 